\title{
Interception of malocclusion in the mixed dentition with prefabricated appliances and orofacial myofunctional therapy
}

\begin{abstract}
This article reports the use of Myobrace ${ }^{\circledR}$ appliances, in conjunction with orofacial myofunctional therapy, for the effective early treatment of a developing malocclusion (Class II, division 2 incisor relationship) in the mixed dentition. The Myobrace ${ }^{\circledR}$ appliances used are prefabricated functional appliances with tooth positioning and myofunctional training characteristics. Myofunctional therapy-through the Myobrace Activities ${ }^{\mathrm{TM}}$ protocol-was implemented in this case for the treatment of incorrect oral habits, such as abnormal lip function. The results showed that an early approach in selected cases, taking also into account patient preferences and compliance, is possible and could be considered in treatment planning and relevant future research.
\end{abstract}

Volume 7 Issue 5 - 2017

\section{Kosmas N Chrysopoulos}

Private Pediatric Dental Practice, Greece

Correspondence: Kosmas N Chrysopoulos DDS MClinDent, Private Pediatric Dental Practice, 2 Ap. Pavlou Street, Rhodes 85133, Greece, Tel (+30) 224II 16394, Email kchrysop@kidsdent.gr

Received: July 02, 2017 | Published: July 06, 2017

Keywords: interception, mixed dentition, myobrace, myofunctional therapy

\section{Introduction}

The appropriate timing of orthodontic treatment for various malocclusions has always been a controversial topic amongst oral health professionals. The importance of managing the developing dentition and occlusion and its effect on the well-being of children, and adolescents has been well recognized. Such management includes the recognition, diagnosis, and appropriate treatment of dentofacial abnormalities. ${ }^{1}$ Regarding Class II, division 2malocclusion, it consists of a deep bite and retroclined upper anterior teeth, while it has been associated with hyper tonicity of the lip muscles. ${ }^{2}$ A Cochrane review that looked at orthodontic treatment for children with this type of problem could not provide any evidence-based guidance to recommend or discourage any type of treatment to correct Class II, division 2 malocclusion in children. ${ }^{3}$ Hence, some authors recommend treatment during the mixed dentition, while there has been a report of successful outcome with the use of a prefabricated functional appliance. ${ }^{4}$ Orofacial myofunctional therapy is a growing field in orthodontic treatment and basically includes the treatment of facial muscle imbalances, training of tongue posture and establishing equilibrium between the tongue, lip and the cheek muscles. Specific orofacial dysfunctions have been considered to be important indicators for the early detection of functional abnormalities, causing deviations from normal dentition development. ${ }^{5}$ Furthermore, it has been shown that changes in the dental formation can be influenced by myofunctional therapy even in non-growing patients. ${ }^{6}$ Prefabricated unctional appliances have demonstrated to produce favorable outcomes in children mainly with Class II, division 1 malocclusion. ${ }^{7,8}$ Myobrace ${ }^{\circledR}$ appliances are prefabricated functional appliances with tooth positioning and myofunctional training characteristics, which are used to correct malocclusions in the developing child, in conjunction with myofunctional therapy-through the Myobrace Activities ${ }^{\mathrm{TM}}$ protocol. Thus, the purpose of this article is to present a clinical case with a malocclusion in the mixed dentition, presenting with Class II/division 2 incisor relationships, successfully treated with Myobrace $\AA$ appliances and myofunctional therapy.

\section{Case presentation}

An 8-year-old girl presented to the practice with a mildly crowded dentition, lingually tipped maxillary central and buccally tipped maxillary lateral incisors. Clinical examination revealed a Class II/ division 2 incisor relationship. There was also a bilateral Class I molar relationship, $6 \mathrm{~mm}$ overjet and $5 \mathrm{~mm}$ overbite, in addition to hypertonicity of the lip, cheeks and chin muscles at rest and upon swallowing (Figure 1). The panoramic radiograph revealed the presence of all permanent successor teeth and no pathology (Figure 2 ). The treatment goal was to improve the position of the anterior teeth and the muscular activity of the orofacial muscles. The parents seeked for a treatment option other than fixed appliances. Thus, the treatment proposed was the use of Myobrace for Kids ${ }^{\mathrm{TM}}$, which is an appliance system, designed specifically to correct poor oral habits and function while treating upper and lower tooth/jaw discrepancies. ${ }^{9}$ The patient was instructed to wear the Myobrace ${ }^{\circledR}$ K1 appliance for 1 hour each day and overnight while sleeping, for a period of 4 months. The K1 contributed to initial myofunctional improvement, followed by the $\mathrm{K} 2$ appliance, which provides arch development in addition to continuing habit correction. It features Dynamicore ${ }^{\mathrm{TM}}$ with a Frankel Cage, which assists in developing the arch form and improving the dental alignment. Reevaluation was planned in 6 months of K2 application (Figures 3\&4). Targeted myofunctional therapy-through the Myobrace Activities ${ }^{\mathrm{TM}_{\text {-was }}}$ also initiated from the start of the treatment. The Myobrace Activities ${ }^{\mathrm{TM}}$ App (available to the patient during scheduled clinic visits, as well as at home) is designed to facilitate the patient's interaction with the treatment. Using created multimedia, the app educates patients regarding habit correction and dietary change. It also sets benchmarks for Myobrace appliance use, while regulating the myofunctional "Myobrace Activities" exercises. Habit correction is achieved by reaching the benchmarks for appliance use and exercise effort, set by the Myobrace Activities App and customized by the practitioner for individual patients. ${ }^{10}$ 


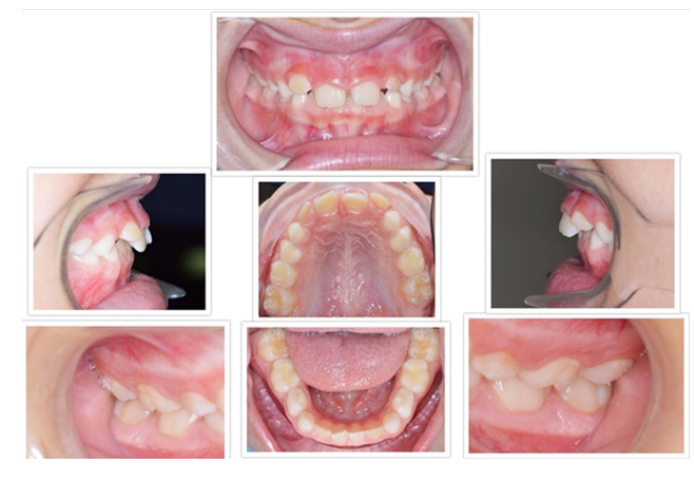

Figure I Pre-treatment intraoral photos.

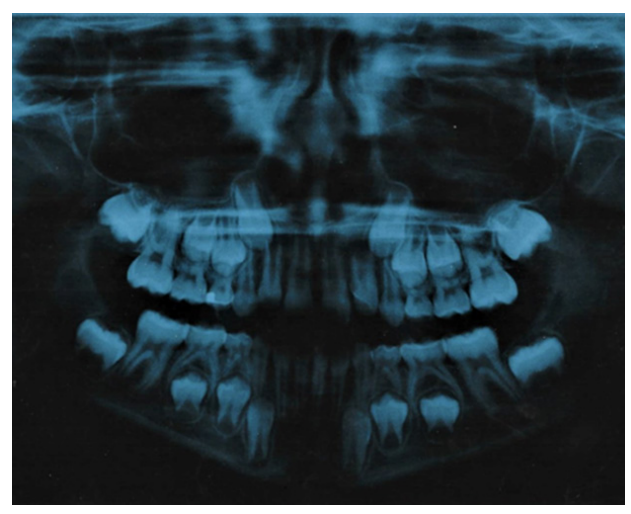

Figure 2 Pre-treatment panoramic radiograph.

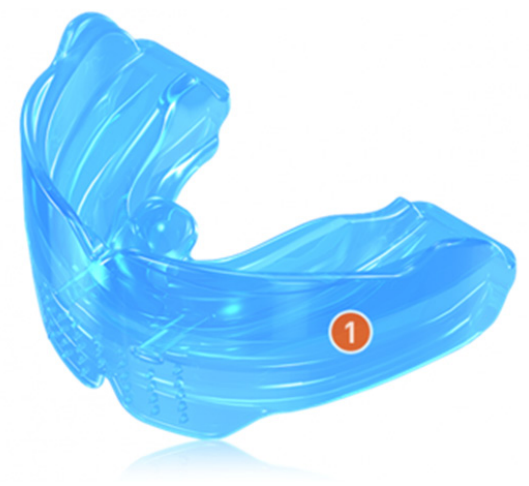

Figure 3 The Myobrace KI appliance.

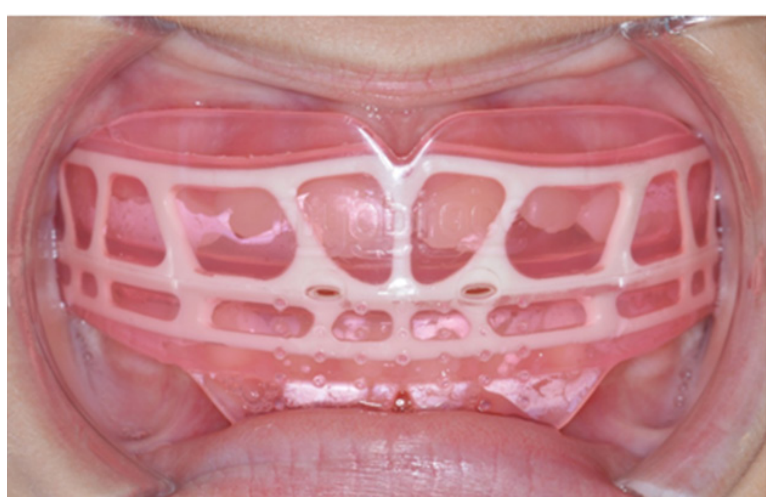

Figure 4 The Myobrace K2 appliance intraorally.

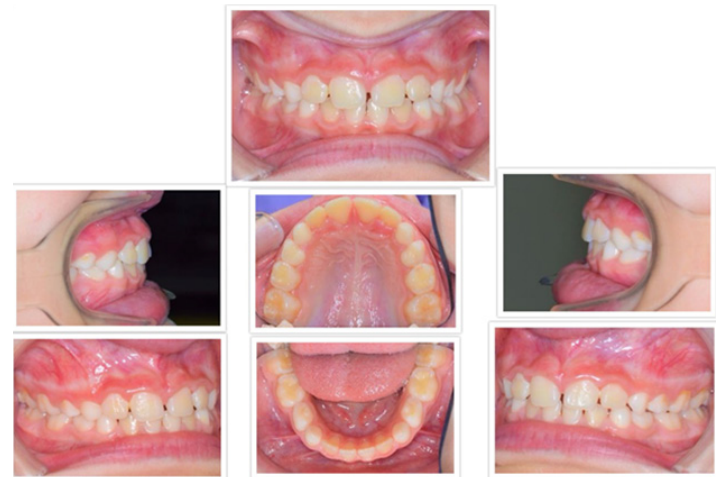

Figure 5 Post-treatment intraoral photos.

\section{Results}

After 10 months of treatment with the K1, K2 and myofunctional therapy, there was a significant improvement in the relationship and position of the anterior teeth, especially the maxillary incisors. There was a reduction in overjet and overbite, both measured to be $2 \mathrm{~mm}$. Moreover, there was less orofacial muscle strain at rest when lips ere together and reduced mentalis and buccinator hyperactivity during swallowing were achieved. Once the patient has gone through the necessary myofunctional exercised to balance even more the orofacial muscular activity, the treatment will be completed (Figure 5).

\section{Discussion}

The clinical case described above shows the successful interception of malocclusion in the mixed dentition, characterized by a Class II/ division 2 incisor relationship and increased overjet and overbite. This was achieved in a relatively short period of time and with minimum discomfort and adverse effects. Ramirez-Yañez \& Faria ${ }^{4}$ reported the early treatment of a Class II/division 2 malocclusion with the Trainer for Kids (T4K), a prefabricated functional appliance. The mandibular advancement and the increase in vertical dimension determined cephalometrically were considered to be a direct effect of treatment with the appliance. In terms of the association between myofunctional disorders and malocclusions, imbalances in the orofacial muscles have been described to negatively affect the developing occlusion. ${ }^{11}$ The Myobrace ${ }^{\circledR}$ appliance, apart from guiding the teeth and promoting their proper alignment, features the following components, enhancing the effectiveness of orofacial myofunctional therapy:

a. Labial and buccal shields: prevent the interposition of lips and cheeks.

b. Tongue tag: acts as a proprioceptive stimulus to the tip of the tongue and as a trainer to correct tongue posture.

c. Tongue guard: prevents tongue thrust and interposition and stimulates nasal breathing

d. Lip bumper: discourages hyperactivity of the mentalis muscle. ${ }^{12}$

Patients with a Class II/division 2 malocclusion have been associated with hyperactivity of the lip muscles and treatment response seems to be better. ${ }^{13}$ The positive outcome in a few months in this case could be due to the fact that malocclusion and imbalance in muscular activity were both targeted in the treatment plan. ${ }^{14}$ Previous studies have shown a positive influence of myofunctional appliance treatment 
on the masticatory and perioral musculature, also when compared to a control group. ${ }^{15-17}$ Compliance with removable orthodontic appliances is always an issue. According to a recent systematic review and meta-analysis, such compliance is considered suboptimal, while patients routinely overestimate duration of wear. ${ }^{18}$ Appropriate patient selection is therefore important. Cooperation may be better before adolescence and it has been suggested that treatment should begin after the age of six and be completed before the onset of puberty. ${ }^{19,20}$ In this clinical case, the patient was 8 years old, daytime use was limited to only 1 hour and the use of the Myobrace Activities App at home for the myofunctional exercises could be monitored at each monthly recall. The anticipated outcome of orofacial myofunctional therapy in conjunction with orthodontic treatment is the reduction of relapse in the long-term. Identifying possible causative factors can contribute to targeted treatment of muscular dysfunction and incorrect posture, as it has been shown for example in anterior open bite cases. ${ }^{21}$ Since orofacial disorders increase the degree of difficulty of orthodontic treatment and contribute to relapse of dentofacial abnormalities, orofacial myofunctional therapy could be a favorable tool to orthodontic treatment. ${ }^{22-24}$ However, there appears to be a scarcity of consistent studies and scientific evidence supporting the use of such therapy in combination with orthodontic treatment to achieve better results. As such, further studies with more rigorous methodology should be carried out to determine the actual effectiveness of myofunctional therapy. ${ }^{25}$ To conclude, this clinical case demonstrates the effectiveness of prefabricated functional appliances (Myobrace ${ }^{\circledR}$ ) appliances, in conjunction with orofacial myofunctional therapy, for the early treatment of a developing malocclusion (Class II/division 2 incisor relationship) in the mixed dentition and could be considered in treatment planning. Prospective clinical studies with adequate sample size and sample selection are needed to assess the combined effect of such appliances with myofunctional exercises and to determine the contribution of each factor to the correction of the developing malocclusion.

\section{Funding}

None.

\section{Acknowledgments}

None.

\section{Conflicts of interests}

The author declares that there is no conflict of interest.

\section{References}

1. American Academy of Pediatric Dentistry. Guideline on Management of the Developing Dentition and Occlusion in Pediatric Dentistry. Clinical practical guidelines. Reference manual. 1990;38(6):253-265.

2. Posen AL. The application of quantitative perioral assessment to orthodontic case analysis and treatment planning. Angle Orthod. 1976;46(2):118-143.

3. Millett DT, Cunningham S, O'Brien KD, et al. Orthodontic treatment for deep bite and retroclined upper front teeth in children. Cochrane Database of Systematic Reviews. 2006;4:CD005972.

4. Ramirez-Yañez GO, Faria P. Early treatment of a Class II, division 2 malocclusion with the Trainer for Kids (T4K): a case report. J Clin Pediatr Dent. 2008;32(4):325-359.

5. Seemann J, Kundt G, Stahl de Castrillon F. Relationship between occlusal findings and orofacial myofunctional status in primary and mixed dentition: part IV: interrelation between space conditions and orofacial dysfunctions. J Orofac Orthop. 2011;72(1):21-32.
6. Moschik CE, Pichelmayer M, Coulson S, et al. Influence of Myofunctional Therapy on Upper Intercanine Distance. J Dent Oral Disord Ther. 2015;3(1):1-7.

7. Usumez S, Uysal T, Sari Z, et al. The effects of early preorthodontic Trainer treatment on Class II, division 1 patients. Angle Orthod. 2004;74:605-659.

8. Ramirez-Yañez G, Sidlauskas A, Junior E, et al. Dimensional changes in dental arches after treatment with a prefabricated functional appliance. $J$ Clin Pediatr Dent. 2007;31(4):279-283.

9. Oscar Quirós Á, Jelsyka Quirós C, Oscar Quirós C. The Secrets of the Trainer and Myobrace Appliances and the Biofunctional System. Amolca, 2016. p. 328.

10. Farrell C. The Myobrace ${ }^{\circledR}$ System: Biologically focused treatment innovation. Australasian Dental Practice. 2016. 74-78 p.

11. Bakke M, Moller E. Occlusion, malocclusion and craniomandibular function. In: Current controversies in orthodontics. Quintessence, USA, 1991. p. 77-102.

12. Aggarwal I, Wadhawan M, Dhir V. Myobraces: say no to traditional braces. Int J Oral Care Res. 2016;4(1):82-85.

13. Posen AL. The application of quantitative perioral assessment to orthodontic case analysis and treating planning. Angle Orthod. 1976;46(2):118-143.

14. Ramirez-Yañez GO, Farrell C. Soft tissue dysfunction: A missing clue when treating malocclusions. Int J Jaw Func Orthop. 2005;1:483-494.

15. Uysal T, Yagci A, Kara S, et al. Influence of pre-orthodontic trainer treatment on the perioral and masticatory muscles in patients with Class II division 1 malocclusion. Eur J Orthod. 2012;34(1):96-101.

16. Yagci A, Uysal T, Kara S, et al. The effects of myofunctional appliance treatment on the perioral and masticatory muscles in Class II, Division 1 patients. World J Orthod. 2010;11(2):117-122.

17. Schievano D, Rontani RMP, Bérzin F. Influence of myofunctional therapy on the perioral muscles. Clinical and electromyographic evaluations. $J$ of Oral Rehabilitation. 1999;26(7):564-569.

18. Al-Moghrabi D, Salazar FC, Pandis N, et al. Compliance with removable orthodontic appliances and adjuncts: A systematic review and metaanalysis. Am J Orthod Dentofacial Orthop. 2017;152(1):17-32.

19. Southard KA, Tolley EA, Arheart KL, et al. Application of the Millon Adolescent Personality Inventory in evaluating orthodontic compliance. Am J Orthod Dentofacial Orthop. 1991;100(6):553-561.

20. Tung AW, Kiyak HA. Psychological influences on the timing of orthodontic treatment. Am J Orthod Dentofacial Orthop. 1998;113(1):29-39.

21. Smithpeter J, Covell D. Relapse of anterior open bites treated with orthodontic appliances with and without orofacial myofunctional therapy. Am J Orthod Dentofacial Orthop. 2010;137(5):605-614.

22. Klocke A, Korbmacher H, Kahl-Nieke B. Influence of orthodontic appliances on myofunctional therapy. J Orofac Orthop. 2000;61(6):414 420.

23. Ohono T, Yogosawa F, Nakamura K. An approach to open bite cases with tongue thrusting habits with reference to habit appliances and myofunctional therapy as viewed from an orthodontic standpoint. Int $J$ Orofacial Myology. 1982;7:3-10.

24. Toronto AS. Long-term effectiveness of oral myotherapy. Int J Oral Myol. 1975;1(4):132-136.

25. Homem MA, Vieira-Andrade RG, Falci SGM, et al. Effectiveness of orofacial myofunctional therapy in orthodontic patients: A systematic review. Dental Press J Orthod. 2014;19(4):94-99. 